Les très grandes infrastructures

de recherche (TGIR) ont acquis

une importance croissante ces

dernières dizaines d'années.

Conçues au départ pour

la physique, elles sont maintenant

utilisées par une communauté

scientifique de plus en plus large,

incluant la chimie, les sciences

de la Terre, la biologie...

Non seulement elles répondent

à des questions scientifiques

essentielles, mais aussi elles

améliorent la compétitivité

des économies (par les défis

technologiques à vaincre

et par les retombées de la recherche

de base), et jouent un rôle important

dans la cohésion européenne.

Dans cet article, Danièle Hulin

présente la " feuille de route »

française des TGIR, publiée

en 2008 en cohérence avec la

roadmap européenne.

\title{
La feuille de route des très grandes infrastructures de recherche
}

Danièle Hulin (daniele.hulin@recherche.gouv.fr)

Directrice de recherche au CNRS, Palaiseau, Chargée de mission à la Direction Générale de la Recherche et de l'Innovation (MESR)

Les très grandes infrastructures de recherche (TGIR) suscitent parfois des réactions contradictoires :

$\checkmark$ d'une part, elles sont indispensables dans le paysage de la recherche scientifique. Que seraient la physique des particules sans le CERN, ou l'astrophysique sans les très grands télescopes qui sondent le fond de l'univers ou traquent les exoplanètes ? Les TGIR résultent aussi de l'évolution de l'instrumentation, qui devient de plus en plus complexe et coûteuse et cesse d'être à l'échelle d'un seul laboratoire, d'où une mutualisation sur de gros instruments ;

$\checkmark$ d'autre part, elles nécessitent des budgets importants et sont parfois regardées avec suspicion par les communautés qui n'en ont pas besoin. Il faut donc examiner avec soin ce qui est vraiment nécessaire au progrès des connaissances et prévoir sur le long terme les financements qui devront être mis en place.

C'est dans cette optique qu'en 2007 François Goulard, alors ministre en charge de la recherche, a décidé d'établir une feuille de route des très grandes infrastructures de recherche, ce qui n'existait pas alors. Cette décision allait dans le sens d'un mouvement d'ensemble des pays européens, stimulés par la publication en 2006 de la roadmap des projets de TGIR en Europe par l'ESFRI, le forum stratégique européen pour les infrastructures de recherche (http://cordis.europa.eu/esfri/).

Quatre groupes de travail ont été réunis, constitués de scientifiques connaissant bien les communautés utilisatrices ou concernées. Leur première tâche a été de recenser toutes les infrastructures susceptibles d'être considérées comme TGIR, existantes, décidées ou souhaitées. À la lumière d'une grille de critères adaptés à chaque discipline scientifique, ils ont sélectionné celles qui pouvaient vraiment entrer dans cette catégorie. Ils ont également débattu des priorités pour les futures infrastructures. Plus de détails sur cette procédure peuvent être trouvés sur le site de travail www.roadmaptgi.fr.

Après différentes consultations et discussions, le ministère a publié en décembre 2008 sa feuille de route des TGIR. Elle peut être téléchargée sur le site web du ministère ou sur le site de travail. Ce document va maintenant servir de base pour une discussion élargie à l'ensemble de la communauté des chercheurs, aux différents conseils et aux citoyens par l'intermédiaire d'instances comme l'Office parlementaire des choix scientifiques et techniques (OPECST). Cette feuille de route n'est pas un document figé, et sera réactualisée régulièrement.

\section{Que peut-on tirer de cet exercice ?}

- Cette feuille de route recense 46 TGIR existantes (dont 30 en physique, voir liste p. 32), auxquelles la France contribue financièrement. Le secteur spatial n'est pas pris en compte dans le document. Tous les domaines sont couverts, y compris la santé, l'environnement et les sciences humaines et sociales (SHS). Les budgets annuels peuvent être très différents selon les secteurs : $107 \mathrm{M} €$ pour le

* Ce point complique d'ailleurs les études bibliométriques, mais il a quand même été relevé qu'une part sensible des publications françaises dans des revues à facteur d'impact élevé provient de travaux effectués grâce à une grande infrastructure.

$\star \star$ Les budgets payés par la France pour le fonctionnement des TGIR existantes $-y$ compris les personnels et le renouvellement usuel des instruments - sont de $21 \mathrm{M} €$ pour le secteur de l'Univers vu de la Terre, $161 \mathrm{M} €$ pour celui des particules et noyaux, $185 \mathrm{M} €$ pour celui de la matière et $70 \mathrm{M} €$ pour le calcul qui sert partiellement à la physique.

\section{Définition d'une TGlR}

Une très grande infrastructure de recherche est un outil établi en vue de mener une recherche propre d'importance et pouvant assurer une mission de service pour une ou plusieurs communautés scientifiques de grande taille. Son coût de construction et d'exploitation est tel qu'il justifie un processus de décision et de financement concertés au niveau national, et éventuellement européen ou international, et une programmation pluriannuelle. Sa gouvernance est centralisée et ses orientations et son évaluation sont assurées par des comités scientifiques de haut niveau. Son accès est ouvert à tous sur la base de l'excellence scientifique. 


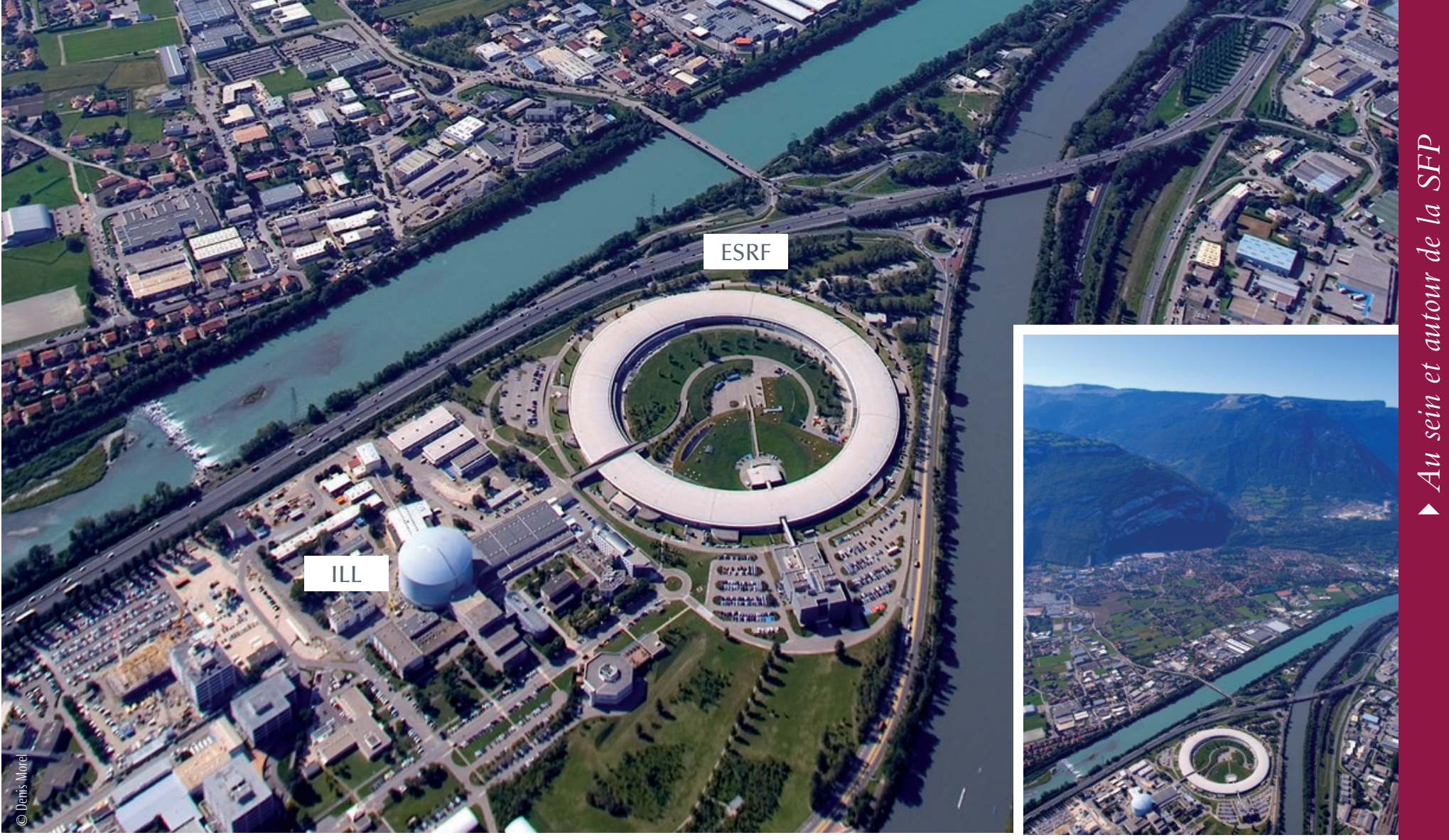

1. Vue aérienne du site de I'ESRF-ILL-EMBL à Grenoble, à la confluence du Drac et de l'Isère. L'ESRF (European Synchrotron Radiation Facility), en activité depuis 20 ans, est l'exemple même d'une très grande infrastructure construite en France par plusieurs pays qui en assurent aussi la gestion. Elle accueille des chercheurs européens pour des projets sélectionnés par des comités de programme aussi bien en physique, en chimie qu'en biologie. Sur la même photo, on voit également l'ILL (Institut Laue Langevin) qui est une TGIR européenne pour l'utilisation des neutrons.

LHC (grand collisionneur de hadrons au CERN) contre 1-2 M€ pour une TGIR en SHS (enquêtes, bases de données, bibliothèques numériques).

- Au-delà des TGIR existantes, il existe deux autres catégories : les TGIR décidées, au nombre de 19, et les TGIR en projet, sélectionnées comme prioritaires. Les premières correspondent à celles pour lesquelles un financement est déjà recherché ou à des entités distribuées en cours de coordination (i. e. les éléments de base existent et une structure de gouvernance adéquate reste à mettre en place). Les TGIR en projet sont des infrastructures dont la conception est bien avancée mais dont le financement n'est pas décidé, ou des projets à plus long terme soumis à la levée de verrous technologiques ou dont la structuration est encore à l'état d'ébauche.

- Le «I " de TGIR est pour " infrastructure " et non pas pour "instrument". Les infrastructures peuvent être localisées sur un seul site (exemple : un synchrotron), distribuées sur plusieurs sites, ou virtuelles (exemple : les bases de données). La notion de réseau de plates-formes a été acceptée, en particulier pour les domaines de la santé et de l'environnement, à condition toutefois que le critère de gouvernance centralisée et de portail unique soit respecté. En physique, on a recensé le cas des plates-formes pour les nanotechnologies, la RMN à très haut champ (commune avec la chimie et la biologie) et les souffleries. Cet inventaire n'en est qu'à son début.

- Dans les propositions de nouvelles infrastructures, une grande partie correspond à des projets européens retenus dans la roadmap d'ESFRI. Cela confirme l'importance des coopérations internationales pour la communauté scientifique française, tout en soulignant l'articulation croissante des politiques de recherche nationale et européenne. Réciproquement, les projets ESFRI présentent dans leur majorité un intérêt pour une ou plusieurs communautés scientifiques nationales. La couverture de toutes les disciplines par la France montre le large spectre de la recherche dans notre pays.

Déjà pour l'existant et de plus en plus pour les projets, les très grandes infrastructures de recherche sont des outils le plus souvent partagés par plusieurs pays et utilisés par des équipes plurinationales ${ }^{\star}$. La mutualisation des financements permet à l'Europe de continuer à programmer des infrastructures qui seront à la pointe de la technologie et apporteront des résultats qui ne pourront être obtenus autrement.
Sans ignorer leur coût ${ }^{\star}$ qui nous impose une programmation et une gestion rigoureuses, les TGIR ont leur place à côté des autres moyens de la recherche. Elles sont aussi des emblèmes visibles qui doivent pouvoir renforcer vis-à-vis des jeunes le prestige et l'attractivité de la science. Enfin, il reste à noter en conclusion que le paysage des infrastructures de recherche a beaucoup évolué, en particulier grâce aux progrès des technologies de l'information et de la communication, et qu'elles sont maintenant des outils au service de toutes les disciplines scientifiques.

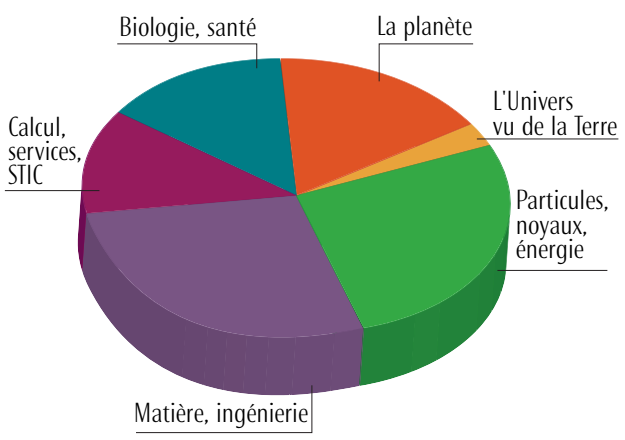

2. Parts financières de la France dans les budgets de fonctionnement - y compris les personnels et le renouvellement usuel des instruments - des TGIR classées en différents secteurs. 


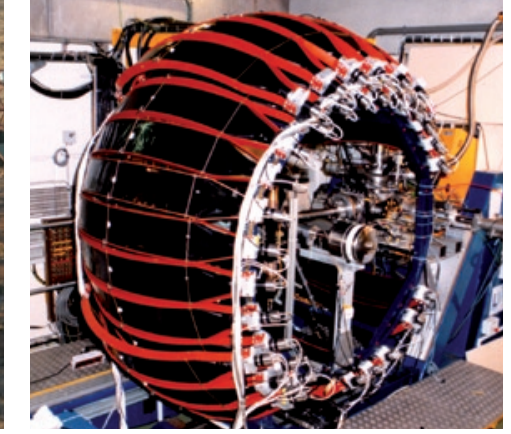

1. Deux des quatre télescopes de HESS.

2. Le détecteur TONNERRE au GANIL.

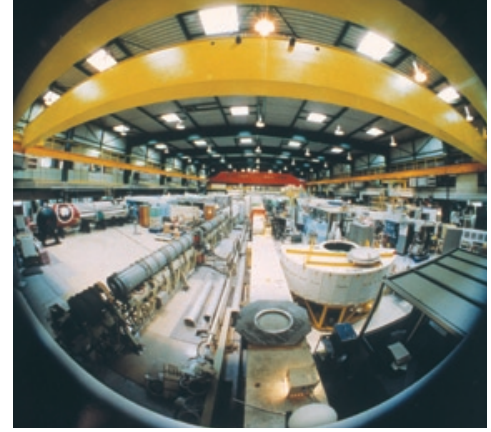

3. Le hall des guides de neutrons du LLB.

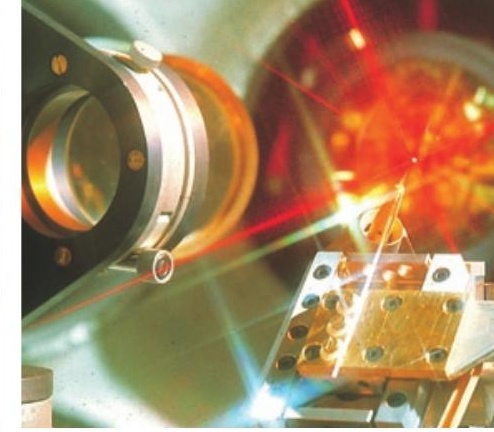

4. La cible du laser-plasma au LULI. $\gg$

\section{Les TGIR existantes}

\section{- L'Univers vu de la Terre}

VLT-I (ESO) ........ Very large telescope (4 grands télescopes de $8 \mathrm{~m}$ pouvant travailler en réseau) - Chili

CFHT ........... Observatoire Canada-France-Hawaii - Hawaii

HESS / HESS II ..... High Energy Stereoscopic System / Télescopes pour imager le rayonnement Cerenkov des rayons gamma dans l'atmosphère - Namibie

IRAM ........... Institut de Radioastronomie Millimétrique

ALMA........... Réseaux de 64 radiotélescopes (domaine submillimétrique) - Chili

LOFAR ........... LOw Freq ARray - Pays-Bas, Allemagne, France et UK CDS ........... Centre de Données astronomiques de Strasbourg

\section{- Particules, noyaux}

CERN, LHC, ...... Organisation européenne pour la recherche détecteurs nucléaire - Large Hadron Collider - près de Genève VIRGO / EGO...... Interféromètre pour ondes gravitationnelles - Italie ANTARES ........ Observation sous-marine de neutrinos de haute énergie - Mer Méditerranée au large de Toulon

GANIL........... Grand Accélérateur National d'Ions Lourds - Caen

JET. ........... Tokamak (instrument destiné à étudier les plasmas de fusion par confinement magnétique) - Royaume-Uni

TORE SUPRA ...... Tokamak à bobines supraconductrices - Cadarache

\section{- La matière}

ESRF ........... European Synchrotron Radiation Facility - Grenoble

SOLEIL ......... Source Optimisée de Lumière d'Énergie Intermédiaire du LURE / Synchrotron - Saclay

LULI ........... Laboratoire d'Utilisation des Lasers Intenses - Palaiseau LIL............. Ligne d'Intégration Laser - près de Bordeaux

LLB ............ Laboratoire Léon Brillouin / Diffusion de neutrons - Saclay ILL............ Institut Laue-Langevin / Infrastructure européenne pour la diffusion de neutrons - Grenoble

LCMI............ Laboratoire des Champs Magnétiques Intenses - Grenoble

LNCMP.......... Laboratoire National des Champs Magnétiques Pulsés - Toulouse

Centrales nanos .... Réseau national de centrales de nanotechnologie RMN ............ Réseau des installations de Résonance Magnétique à très hauts champs ... Nucléaire à très hauts champs

Souffleries........ Souffleries pour l'aéronautique et le spatial

\section{Calcul, services..}

GENCI........... Grand Équipement National pour le Calcul Intensif

CINES ........... Centre Informatique National de l'Enseignement Supérieur - Montpellier

IDRIS .......... Institut de Développement des Ressources en Informatique Scientifique - Orsay

CCRT............ Centre de Calcul Recherche et Technologie au CEA

CC-IN2P3 ......... Centre de Calcul IN2P3-IRFU - Lyon

RENATER ........ Réseau National de Télécommunications pour la Technologie, l'Enseignement et la Recherche

\section{Les TGliR du futur, proche ou plus lointain}

\section{Les décidées}

RJH........... Réacteur d'irradiation Jules Horowitz - Cadarache

ITER ............ International Thermonuclear Experimental Reactor - Cadarache

(IFMIF) EVEDA ..... International Fusion Materials Irradiation Facility / Engineering Validation Engineering Design Activities - Japon et Europe

SPIRAL $2 \ldots \ldots$.... Système de production d'Ions Radioactifs en Ligne (au GANIL) - Caen

FAIR .......... Facility for Antiproton and Ion Research (participation) - Darmstadt

XFEL $\ldots \ldots \ldots \ldots$ X-ray Free Electron Laser (participation) - Hambourg

ILE ............. Institut de la Lumière Extrême - Palaiseau

PETAL............ PETawatt Aquitaine Laser (financement encore incomplet). Élément du projet européen HiPER

ILL - upgrade 1 .... Millenium - Upgrade de I'Institut Laue Langevin : phase 1 - Grenoble

ESRF - upgrade 1.... Upgrade de l'European Synchrotron Radiation Facility : phase 1 - Grenoble

\section{- Les hautement prioritaires}

E-ELT ........... Extremely Large Telescope (42 m) pour l'Europe

CTA $\ldots \ldots \ldots \ldots$ Cherenkov Telescope Array facility (projet international) - Suite de HESS 2

ESRF - upgrade 2 ... Upgrade de l'European Synchrotron Radiation Facility : phase 2

ILL - upgrade 2.... Upgrade de I'Institut Laue Langevin : phase 2 Magnétisme ....... Haut champ magnétique pour ESRF et ILL - Grenoble PRACE ........... Partnership for Advanced Computing in Europe

\section{- Les prioritaires}

LSST ........... Large Synoptic Survey Telescope ; imagerie grand champ - Chili

SKA ........... Square Kilometer Array (radiotélescope géant)

Km3 Net ........ Observation sous-marine de neutrinos de haute énergie

Super LHC........ Upgrade du LHC. La décision dépend des résultats du LHC

ILC ou CLIC........ Effort global en vue d'un collisionneur linéaire mondial pour la physique des particules

ULISSE / LSM ...... Agrandissement du Laboratoire Souterrain de Modane (physique des particules)

Eurisol ........... Accélérateur de faisceaux d'ions radioactifs MYRRHA......... Réacteur nucléaire de recherche - Belgique IFMIF ........... International Fusion Materials Irradiation Facility ELI ............ Extreme Light Infrastructure

ESS .............. European Spallation Source (source de neutrons)

EMFL .......... Réseau distribué de 4 centres de champs magnétiques intenses en Europe

Liste des très grandes infrastructures de recherche en physique, existantes ou à venir, sélectionnées dans le cadre de la feuille de route française (d'après le document publié le 9 décembre 2008 par le ministère de l'Enseignement supérieur et de la Recherche). 